\title{
The Determination of Crystallographic Textures from Selected Areas of a Specimen by Electron Diffraction
}

\section{F. J. HUMPHREYS}

Department of Metallurgy and Materials, Imperial College, London SW7 2BP

(Received February 2, 1983)

A technique for the determination of partial pole figures with an angular resolution of $<3^{\circ}$, from selected areas of a thin foil, is described. A microcomputer, interfaced to an unmodified JEOL $100 \mathrm{CX}$ TEMSCAN electron microscope is used to scan a diffraction pattern over a detector, tilt the specimen in steps of $1.5^{\circ}$ over a range of $\pm 50^{\circ}$, and plot the resulting data as a semiquantitative pole figure. The application of the technique to the study of materials which deform inhomogeneously is discussed, and examples are given of pole figures obtained from deformed single phase and two phase aluminium specimens.

\section{INTRODUCTION}

There are many techniques now available for the study of crystallographic orientation in materials. Selected area and microbeam electron diffraction methods are suitable for determining the orientation of a small area of a thin foil, and electron backscattered and channelling patterns yield similar information from thick specimens.

There are however, instances in which it is desirable to study the orientation of a small area, less than $\sim 50 \mu \mathrm{m}$ in diameter, in which there are significant orientation changes, and in which the coherent scattering unit is less than $\sim 1 \mu \mathrm{m}$ in diameter. For example, in deformed 
metals, regions of inhomogeneous deformation, such as shear bands, or the areas adjacent to grain boundaries or second-phase particles are of interest.

In these instances, none of the techniques mentioned above is suitable, because, even if the spatial resolution is not a limiting factor, as for the SAD or ECP methods, the number of individual diffraction patterns which are required for a detailed investigation is too great. For example, the study of a $10 \mu \mathrm{m} \times 10 \mu \mathrm{m}$ area of a thin foil with a subgrain size of $0.5 \mu \mathrm{m}$ would require the analysis of $\sim 400$ diffraction patterns. In order to carry out such an investigation, a technique is required which combines the spatial resolution of the electron microscope methods with the ability of the X-ray texture method to deal with multiple orientations.

This paper describes a technique whereby semi-quantitative partial pole figures may be determined rapidly and automatically from selected areas of a thin foil, using a transmission electron microscope controlled by a computer.

\section{PRINCIPLE}

The technique is geometrically rather similar to the conventional X-ray transmission texture method. (See e.g. Cullity, 1978; Hatherly and Hutchinson, 1979.)

Consider an electron diffraction pattern from a selected area of a thin foil which has a range of orientations, as in Figure 1a. Reference directions $\mathrm{A}, \mathrm{B}$ and $\mathrm{C}$ in the plane of the specimen are marked, and the electron beam is incident normally on the specimen (D). The distribution of diffracted intensity around the $h k l$ Debye ring is a measure of the distribution of the normals to those $h \mathrm{kl}$ planes which are inclined at the Bragg angle to the beam. As the Bragg angles are generally less than $1^{\circ}$, these data fall on the edge of the $h \mathrm{kl}$ pole figure shown in Figure $1 \mathrm{~b}$. If the specimen is now tilted through an angle $\Delta \alpha$ about the axis $\mathrm{AB}$, and the intensity of the Debye ring from $\delta=0$ to $180^{\circ}$ is measured, then this information may be plotted on the pole figure along the great circle AEB (Figure 1c). Thus by repeating the experiment a number of times with tilts of up to $\pm \alpha$, the partial pole figure of Figure 1d is obtained.

An alternative representation of the data is to plot the pole figure for the plane normal to $\mathrm{C}$, as in Figure 1e. This brings the two segments 


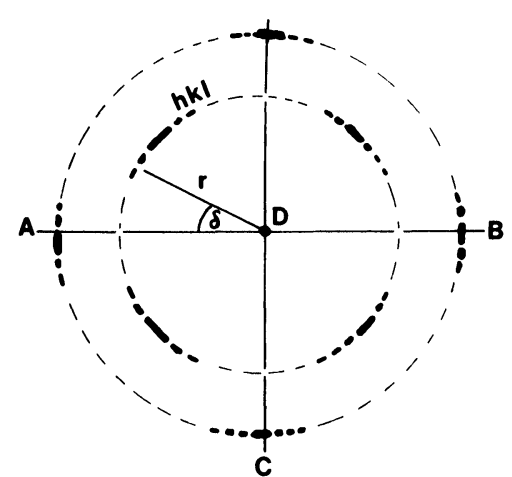

(a)

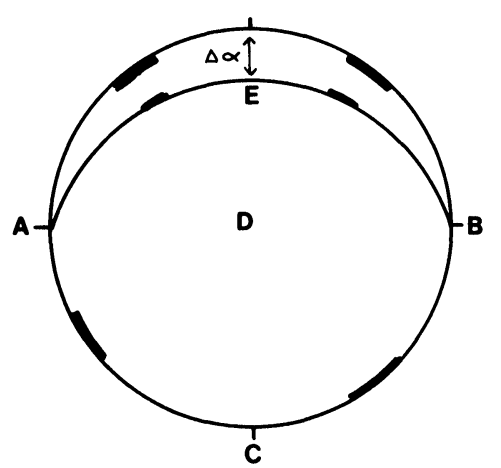

(c)

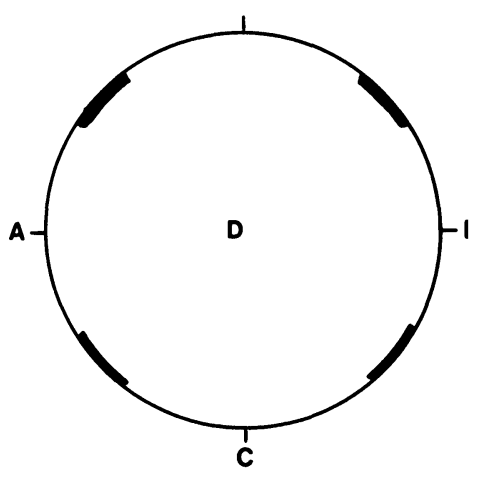

(b)

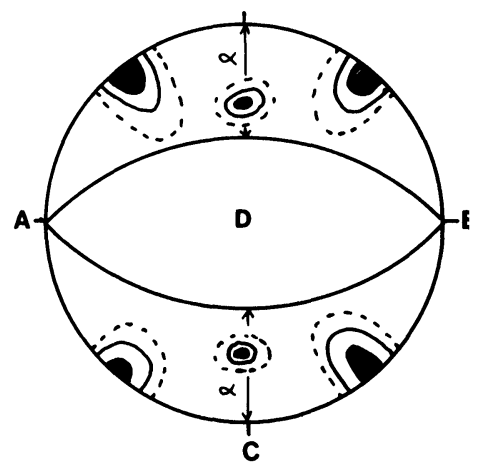

(d)

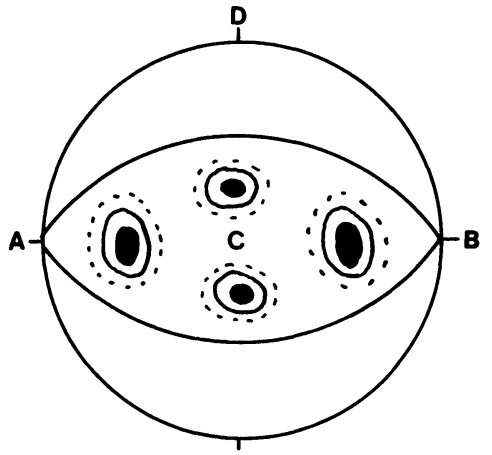

\section{FIGURE 1}

a) Schematic diffraction pattern.

(e)

b) Data from (a) plotted on $h \mathrm{kl}$ pole figure.

c) Pole figure including data from specimen tilted $\Delta \alpha$ about axis $\mathrm{AB}$.

d) Partial pole figure obtained by tilting the specimen through $\pm \alpha$.

e) The same data replotted as a pole figure in the plane perpendicular to $\mathrm{C}$. 
together, and this representation has been used throughout the present investigation.

It would not be practicable to carry out such an experiment manually, but the availability of small computers which can readily be programmed to control the entire procedure rapidly and automatically, has enabled this technique to become viable as a routine method of crystallographic analysis.

\section{ACCURACY AND RESOLUTION}

\section{a) The selected area}

The area being examined will change as the specimen is tilted. Lateral movement of the specimen may, with a good eucentric tilting stage be minimised to $\sim 1 \mu \mathrm{m}$, thus setting a lower limit to the area which can be studied.

In addition, as the specimen is tilted through an angle $\alpha$, the selected area of diameter $X$, becomes an ellipse of the major axis $X / \cos \alpha$, and therefore the volume of material contributing to the diffraction pattern increases.

\section{b) Intensity of diffraction}

The basis of the present technique is that the existance of a beam diffracted in a particular direction is indicative of there being a crystallite with a plane of the appropriate orientation, thereby enabling at least a qualitative estimate to be made of the distribution of crystallographic poles.

Although the intensity of the diffracted beam depends on the volume of material which is diffracting, there are so many other factors to be taken into account, such as thickness, crystallite size, deviation from the Bragg angle, absorbtion, multiple scattering and many beam effects (see e.g. Hirsch et al., 1965) that it is not practicable to relate quantitatively the intensity of the diffracted beams to the pole distribution. However, while carrying out the experiment on a particular area, some of these factors do not change greatly, and e.g. preliminary experiments have however shown that within the range of specimen thickness 1000 $\AA$ to $5000 \AA$ in aluminium, the increased absorbtion due to the effective change in thickness on tilting the specimen is balanced by the increased 
volume which is diffracting, and the intensity of diffraction does not change greatly. Thus the intensity of diffraction in a particular Debye ring, from a selected area can be interpreted semiquantitatively in terms of pole distribution.

\section{c) Background intensity}

The diffraction spots are superimposed on a background intensity due to inelastically scattered electrons. For thick specimens, the ratio of elastic to inelastic scattering decreases, thus setting on upper limit to the usable specmen thickness, which for aluminium at $100 \mathrm{KeV}$ is $\sim 5000 \AA$. It is clear that the technique would benefit from the use of a higher accelerating voltage.

The background intensity may not be uniform for a given angle of scattering. For example in Figure 2, where data is being acquired from the $h_{1} k_{1} l_{1}$ Debye ring, the background intensity at point $\mathrm{A}$ will be greater than at point $B$ because of the inelastic scattering associated with the diffraction spot $\mathrm{C}$, on the $h_{2} k_{2} l_{2}$ ring. In a similar way, Kikuchi lines crossing the $h_{1} k_{1} l_{1}$ ring will be detected, and will give rise to spurious results. In order to eliminate these effects, it is necessary to measure the intensity not only of the Debye ring, but also of the background just inside or outside the ring. Subtraction of these two readings giving a much more accurate measure of the intensity of elastic scattering on the Debye ring.

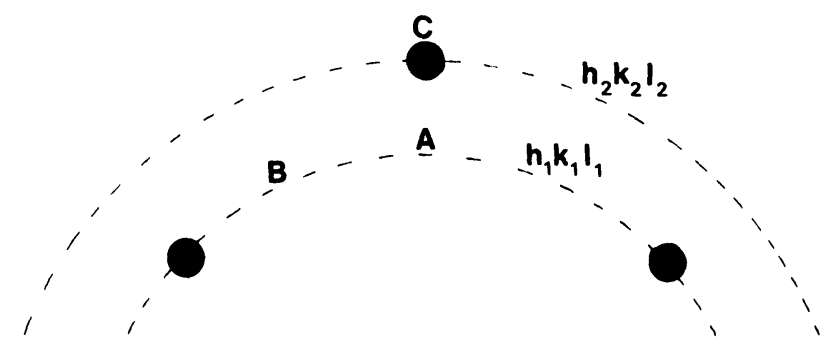

FIGURE 2 The effect of nearby diffraction spots on the background intensity.

\section{d) Resolution}

A crystal of thickness $t$, or a crystallite of this diameter will diffract strongly over an angular range of $\sim d / t$ (Hirsch et al., 1965). Thus for 
crystallites larger than $\sim 100 \AA$, the angular resolution should be better than $1^{\circ}$. This factor will not therefore limit the resolution of the experiment, if very thin regions of the specimen are avoided.

There is also a limitation on the angular resolution around the Debye ring, imposed by the finite size of the detector. If a detector of diameter $P$ is used to scan a Debye ring of radius $r$, then the resolution $(\Delta)$ around the ring is given by:

$$
\Delta=\frac{P}{r}=\frac{P d}{L \lambda}
$$

where $L$ is the camera length, $\lambda$ the wavelength of the electrons, and $d$ is the planar spacing. Thus with the equipment described below, operating at $100 \mathrm{KeV}$ with a camera length of $\sim 380 \mathrm{~cm}$, and a $3 \mathrm{~mm}$ diameter aperture over the detector, the resolution should be $2.9^{\circ}$ for 111 poles and $1.8^{\circ}$ for 220 poles in aluminium.

\section{METHOD}

\section{a) Equipment}

The work was carried out using a JEOL 100 CX (TEMSCAN), a microcomputer, and a simple interface.

The prime requirement is for a transmission electron microscope with the following facilities:

1) Side entry, eucentric tilting stage, motor driven about the rod axis.

2) Transmission electron detector.

3) Pre or post specimen deflection coils.

The requirements for the microcomputer are not critical, although a good graphics capability is desirable.

In the present work, a Research Machines $380 \mathrm{Z}, 56 \mathrm{~K}$ computer with integral 10 bit analogue input/output boards was used.

As the signals between the computer and the microscope are of the order of a few volts, interfacing presented little problem and circuits for switching, amplification, buffering, filtering etc. were readily constructed. 


\section{b) Computer control of the microscope}

The intensity around the Debye ring is measured by scanning the ring across the transmission detector (Figure 3). This is achieved by deflecting the beam in a circle of radius $r_{h k l}$ centred on the detector. This may be carried out either above the specimen using the TEM dark field deflection coils, or below the specimen with STEM dark field deflection coils. The latter method was adopted in the present work. The circular scan was achieved by calculating and storing in the computer, $n$ sets of $\mathrm{x}, \mathrm{y}$ coordinates from a circle, and converting these to analogue voltages which were then applied to the beam deflection coils via operational amplifiers. The number of data points $(n)$ on the circle depends on the resolution required. For the present investigation, steps of $1.5^{\circ}$ were used as this is consistent with the resolution of $2^{\circ}$ to $3^{\circ}$ set by the detector aperture (Section 3d).

Tilting of the specimen was achieved by using the computer to control the tilt motor of the side entry goniometer. Although this is not as precise as using a stepping motor, it was found to be accurate to $1 \%$. The specimen was tilted in steps $(\Delta \alpha)$ of $1.5^{\circ}$ up to a maximum of $50^{\circ}$.

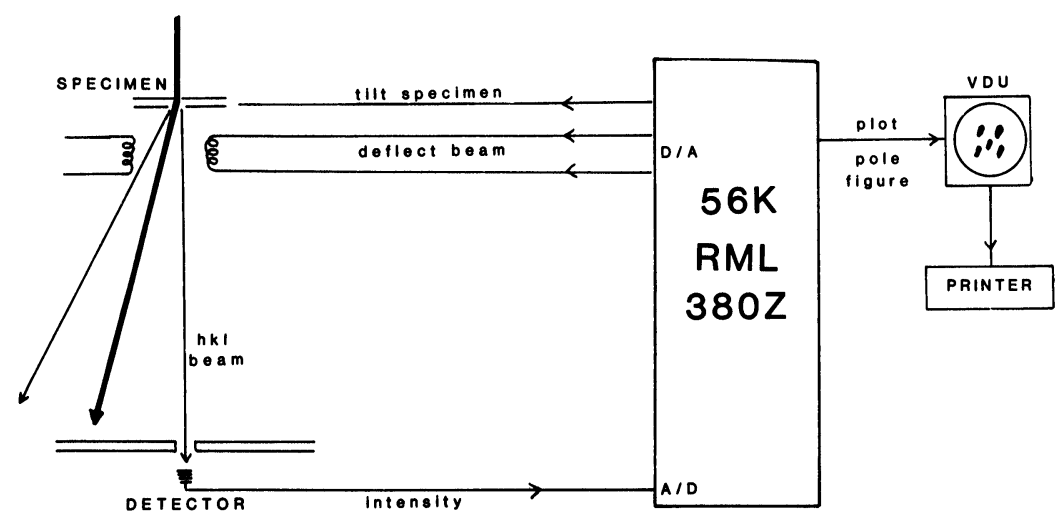

FIGURE 3 Schematic diagram of the equipment.

\section{c) Alignment}

Initial alignment of the system is best carried out with a randomly oriented polycrystalline test specimen, such as an evaporated film, although this is not essential. A fast $(10 \mathrm{~Hz})$ circular scan is applied 
to the beam, and the diffraction pattern is observed on the fluorescent screen. The diameter and circularity of the scan are adjusted by potentiometers until the required Debye ring passes over the centre of the detector aperture.

The current equipment employs three sets of adjustment potentiometers so that the scan may first be aligned for several different Debye rings, and then switched to the selected one, without realignment.

\section{d) Data acquisition}

The transmission electron detector on the JEOL $100 \mathrm{CX}$ has an output voltage range which is compatible with the analogue to digital converter of the $380 \mathrm{Z}$. The contrast and brightness controls of the detector amplifier were adjusted to give a signal within the range $0-5$ volts, and this was digitised to 255 levels. A simple filtering circuit was found to be necessary in order to reduce noise at the low signal levels used.

Each experimental run thus consisted of 120 readings at $1 \frac{1}{2}^{\circ}$ intervals around the Debye ring, and the same number of readings of the adjacent background, the procedure being repeated for 67 tilts at $1.5^{\circ}$ intervals between $+50^{\circ}$ and $-50^{\circ}$. This gave a total of 8040 data points for each Debye ring under investigation.

The data were displayed graphically on the VDU during acquisition, and stored on disc for further analysis.

With the current equipment, the rate limiting factor is the time constant of the detector filter, and an experimental run takes $\sim 15$ minutes. This is much more rapid than X-ray texture methods, and it is planned to substantially reduce this time by modifications to the detector system.

\section{e) Pole figures}

The $380 \mathrm{Z}$ was used to plot the data as pole figures of the type discussed in Section 2. Because of the limited number of subgrains within the selected area of the specimen, the distribution of orientations is generally not sufficiently smooth for computer plotting of contours on the pole figure, except for highly deformed specimens. Pole figures for visual inspection were plotted at a screen resolution of $160 \times 96$ points, at 16 intensity levels, hard copy being obtained on an Anadex DP 9501 dot-matrix printer, and the pole figures of Figures 4, 5 and 7 were obtained in this way. There is some angular distortion in these figures, and for the purposes of measurement, pole figures were plotted for 3 
intensity levels at the higher resolution of $319 \times 192$ points, and printed with a diameter of $30 \mathrm{cms}$.

\section{f) Correlation with the image}

Unlike X-ray texture methods, the present technique allows direct imaging of that part of the specimen which contains a particular component of the texture.

Having acquired a pole figure, which is displayed on the VDU, a particular point on the pole figure is selected by moving a cursor on the screen. Tilt and beam deflection coordinates corresponding to that particular data point are then set by the computer. If an objective aperture is now inserted on the optic axis, and the microscope set to imaging mode, a dark field image, of that region of the specimen contributing to the selected texture component is obtained.

\section{g) Computer programme}

The data acquisition programme was written in BASIC, with machine code subroutines for analogue input and output, and for fast scanning during alignment. The main features of the programme are outlined below in Table I.

TABLE I

Computer Programme

\begin{tabular}{|c|c|c|}
\hline $\begin{array}{l}\text { COORDINATES } \\
\text { Calculated for scan } \\
\text { of Debye ring and } \\
\text { background. }\end{array}$ & $\begin{array}{l}\text { ALIGNMENT } \\
\text { a) Scans for beam } \\
\text { alignment } \\
\text { b) Detector sensitivity } \\
\text { c) Align scan start } \\
\text { with tilt axis. }\end{array}$ & $\begin{array}{l}\text { DATA ACQUISITION } \\
\text { a) Scan diffraction } \\
\text { pattern and background } \\
\text { b) Plot data on VDU } \\
\text { c) Tilt specimen } 1.5^{\circ} \text { and } \\
\text { repeat (a) } \\
\text { d) Save data on disc } \\
\text { e) Image of texture } \\
\text { component. }\end{array}$ \\
\hline
\end{tabular}

\section{RESULTS}

Results are presented below for three types of aluminium specimen, chosen to illustrate potential applications of the technique. 


\section{a) Undeformed}

The texture of a $10 \mu \mathrm{m}$ diameter region of a thin foil of recrystallised aluminium was obtained. Although this is clearly not the simplest way of determining the orientation of an annealed single crystal, it is a sensitive test of the angular resolution of the method.

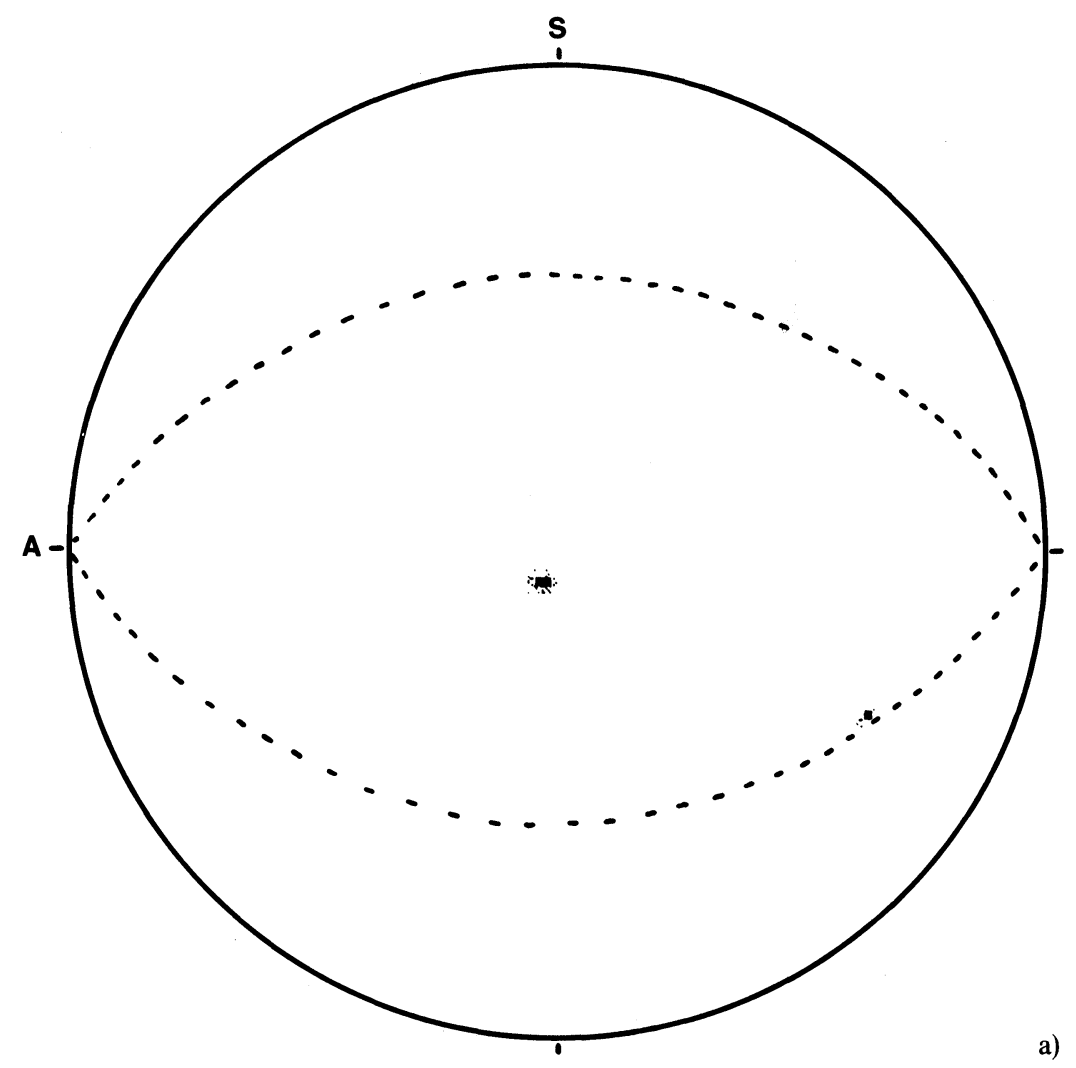

FIGURE 4 Pole figures from undeformed aluminium.

a) 111 .

b) 220 .

The dotted lines show the limits of data acquisition $\left( \pm 50^{\circ}\right)$. Axis of tilt $(\mathrm{A})$ and specimen normal (S) are marked. 
The 111 and 220 pole figures are shown in Figure 4. In both cases, the pole positions can be located to within one data point $\left(1.5^{\circ}\right)$ and scatter about the poles is $\pm 1.5^{\circ}$. This is consistent with the resolution as discussed in Section 3. It should be noted that the data is clean, even in a specimen where variations in background intensity, as discussed in Section $3 c$ are severe.

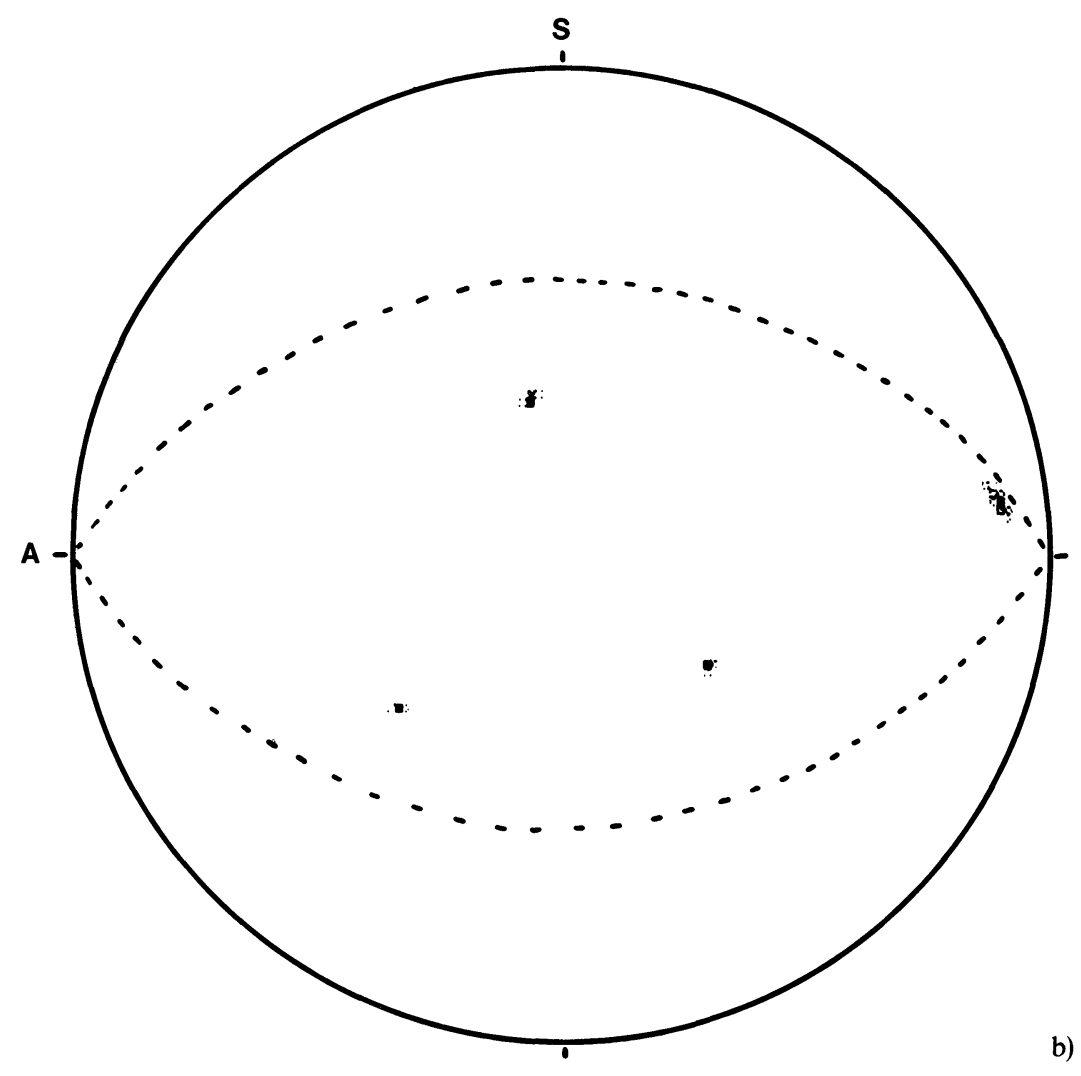




\section{b) Rolled aluminium}

111 and 220 pole figures from a $10 \mu \mathrm{m}$ diameter region within a single grain, of a thin foil of aluminium reduced $80 \%$ by cold rolling are shown in Figure 5. Both the 111 and 220 poles show a spread of orientations of $\sim 20^{\circ}$, the axis of rotation being close to the pole marked $X$ in Figure $5 b$.

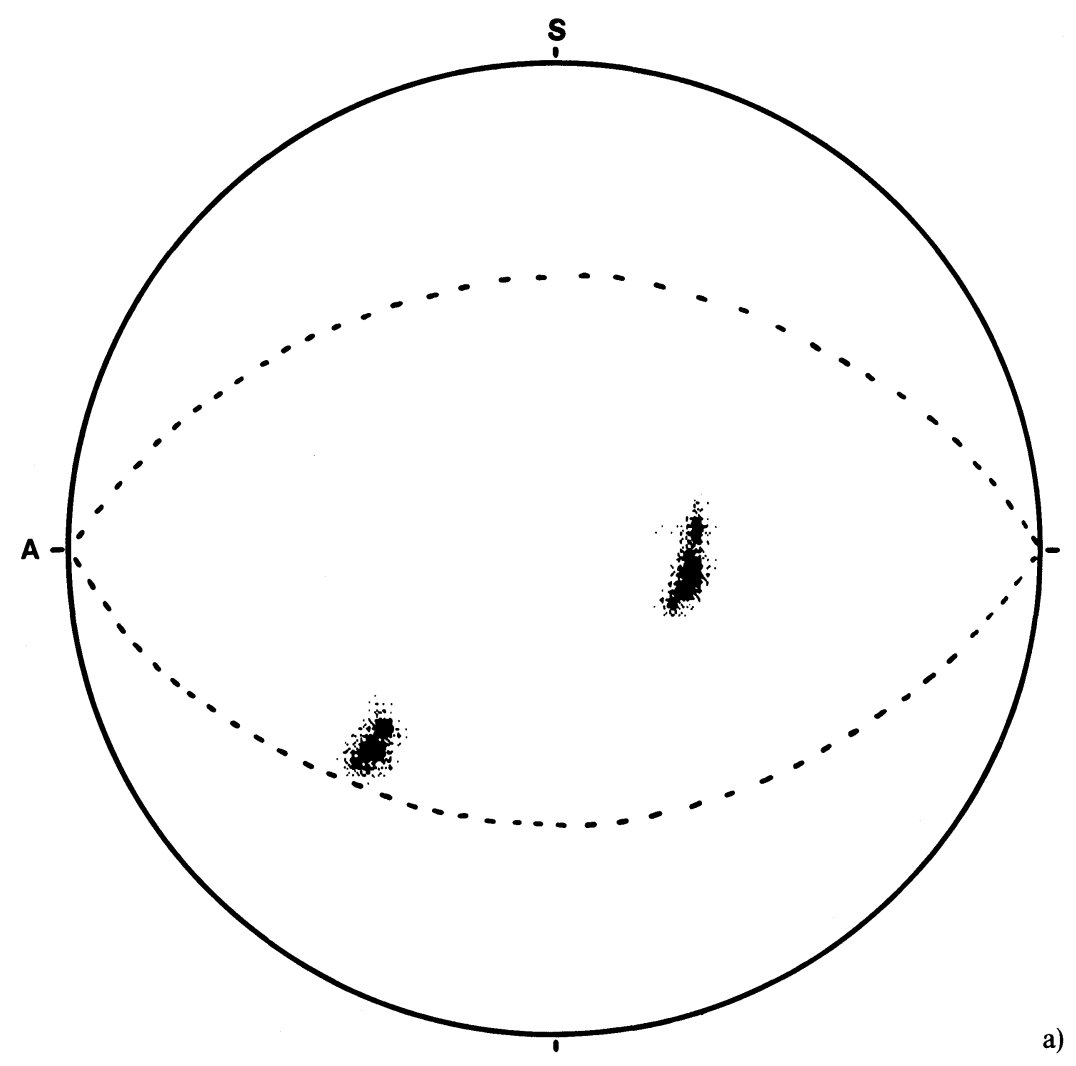

FIGURE 5 Pole figures from aluminium reduced $80 \%$ by cold rolling.

a) 111 .

b) 220 . 


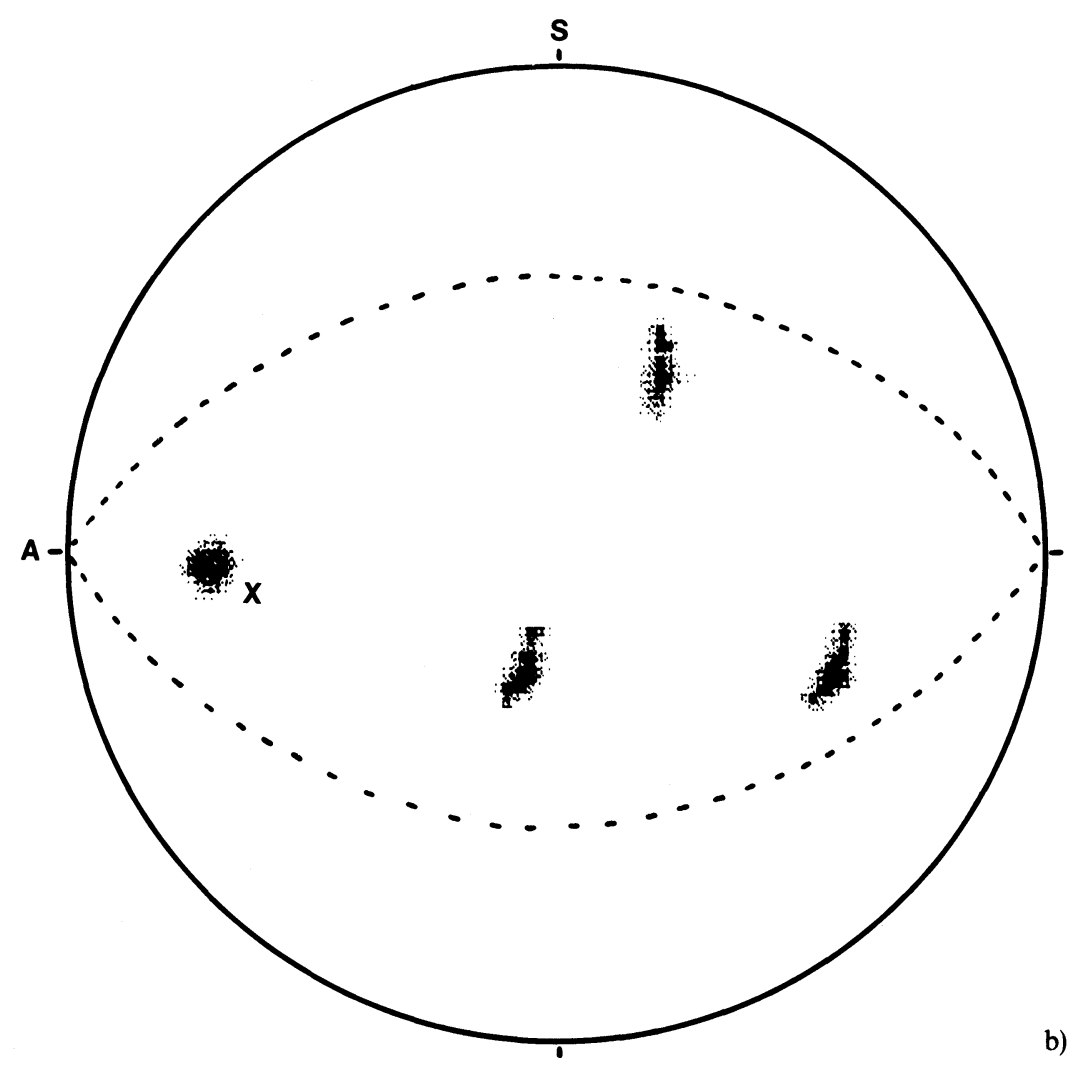




\section{c) Two-phase alloy}

If a metal contains non-deformable particles, then these may alter the deformation pattern of the metal in such a way as to produce zones, adjacent to the particle which are substantially misoriented with respect to the rest of the matrix. These zones may affect not only the deformation of the material, but also its recrystallisation behaviour (Humphreys, 1977).

It is extremely difficult to obtain information about these misorientations by conventional diffraction methods, although Humphreys (1979) has used a dark field imaging method to determine components of the misorientation which have axes in the plane of the specimen.

Specimens from that investigation have now been analysed using the texture method. A thin foil of orientation (101) was prepared from a single crystal of aluminium which had been deformed to a tensile strain

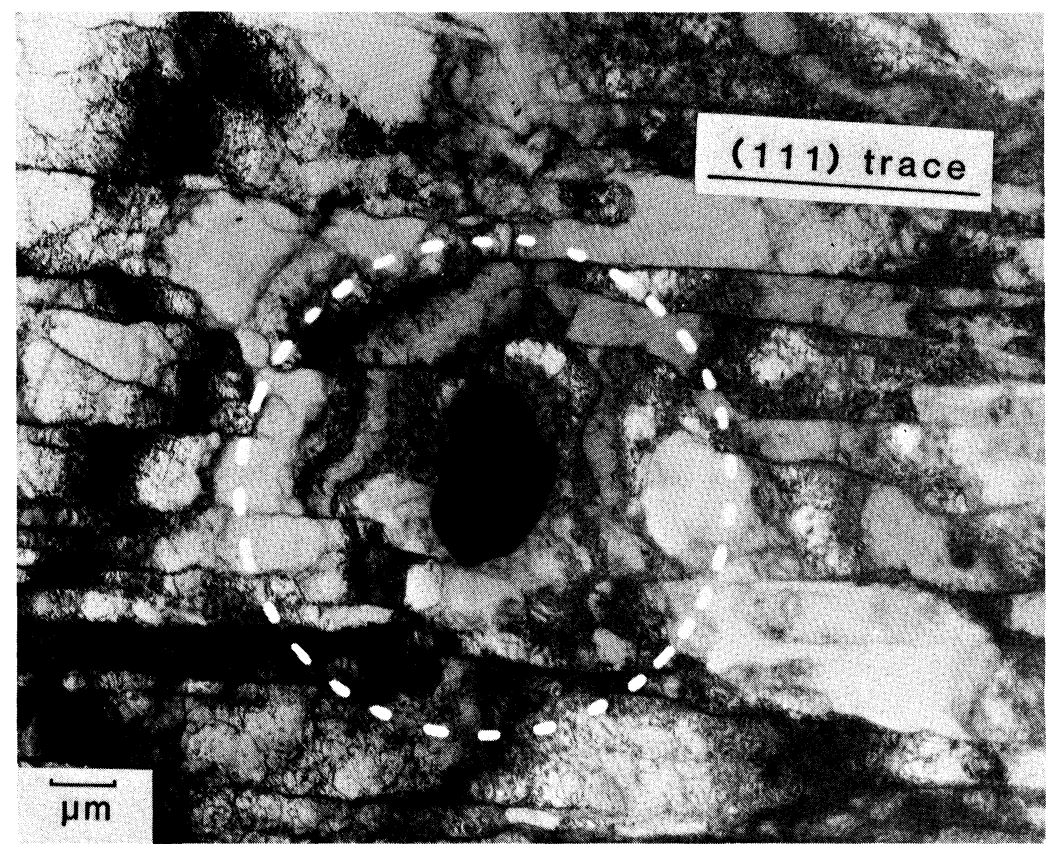

FIGURE 6 Micrograph of a silicon particle in a deformed two-phrase aluminium alloy. There is an alignment of the subgrains parallel to the primary slip plane (111). 
of 0.35 on the primary slip system (111) [101], and which contained particles of silicon of diameter $\sim 3 \mu \mathrm{m}$. Further details of the specimen are given in Humphreys (1979). Figure 6 is a micrograph showing a particle, and the area, of diameter $\sim 7 \mu \mathrm{m}$, which was selected for texture determination. The 111 pole figure for this region is shown in Figure $7 \mathrm{a}$, and a 111 pole figure from an adjacent area, which did not contain a particle, is shown in Figure $7 \mathrm{~b}$. Comparison of the two figures clearly shows the extensive misorientations associated with the particle. Measurement of the pole distribution shows that the axis of misorienta-

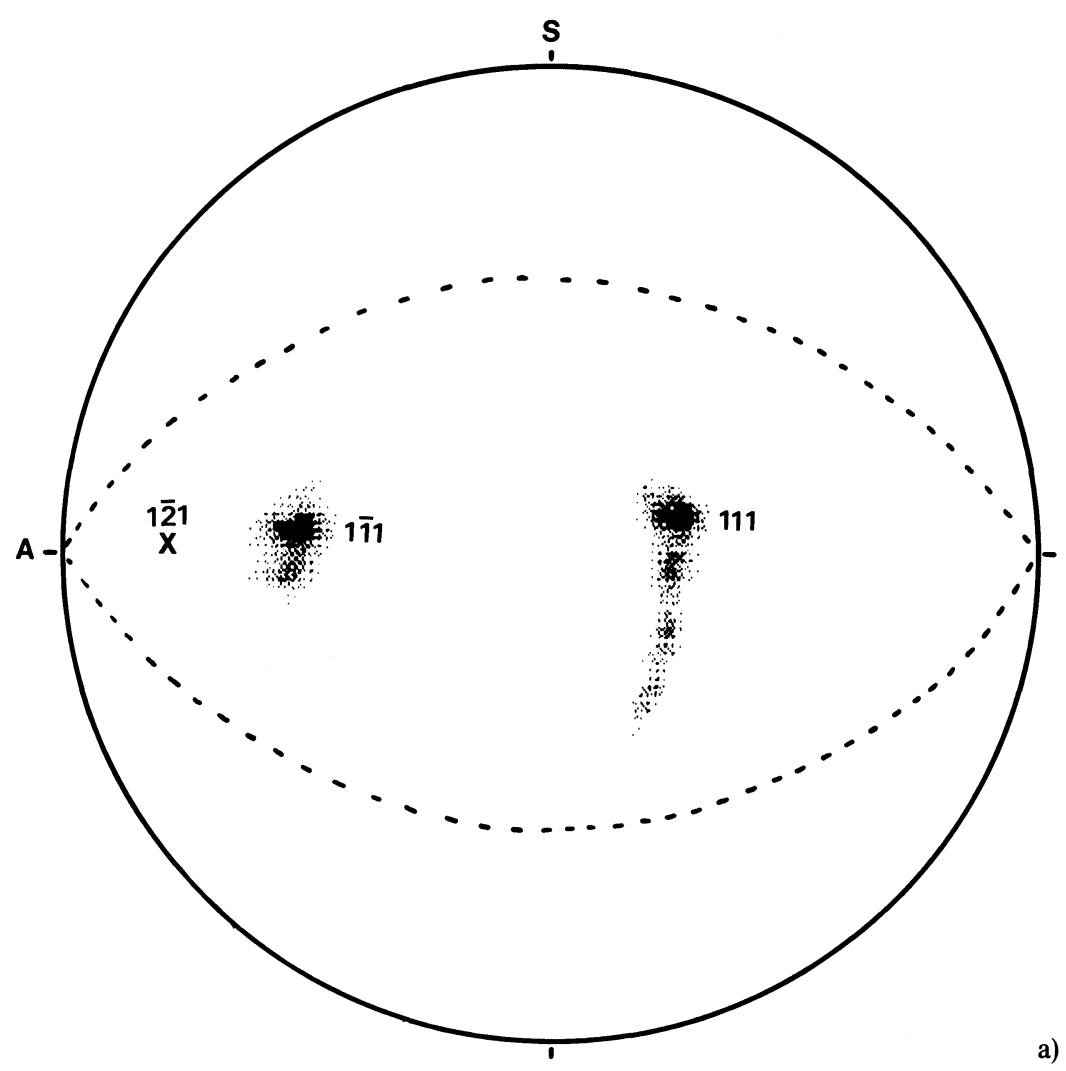

FIGURE 7111 pole figures.

a) Area containing particle, shown in Figure 6.

b) Adjacent area, not containing a particle. 
heterogeneities in deformed materials, the technique may well be of value in the investigation of other materials with fine structures, such as evaporated thin films, splat-quenched metals, or any other fine-grained polycrystalline aggregate.

\section{Acknowledgements}

I would like to thank Dr B. A. Unvala, and Mr D. A. Waters for advice concerning the interfacing of the computer to the microscope. The research was aided by a grant from the Science and Engineering Research Council.

\section{References}

Cullity, B. D. Elements of X-Ray diffraction. Addision-Wesley (1978).

Hatherly, M. and Hutchinson, W. B. An introduction to textures in metals. Institution of Metallurgists. Monograph No. 5 (1979).

Hirsch, P. B., Howie, A., Nicholson, R. B., Pashley, D. W. and Whelan, M. J. Electron Microscopy of thin crystals. Butterworths, London (1965).

Humphreys, F. J. Acta Metall. 25, 1323 (1977).

Humphreys, F. J. Acta Metall. 27, 1801 (1979). 